\title{
AdeQuaS: Ferramenta Fuzzy para Avaliação da Qualidade de Software
}

\author{
Kelly Rejane de Oliveira \\ (kellyr@tutopia.com.br) \\ Arnaldo Dias Belchior \\ (belchior@unifor.br) \\ Mestrado em Informática Aplicada - MIA \\ Universidade de Fortaleza - UNIFOR \\ Av Washington Soares, 1321 \\ 60.811.341 - Fortaleza - CE - Brasil
}

\begin{abstract}
Resumo
A qualidade de software tem tido uma importância crescente como fator crucial de competitividade das organizações. A avaliação da qualidade de software é imprescindível na obtenção e garantia da qualidade tanto no processo de desenvolvimento de software quanto no produto final.

Este artigo apresenta a ferramenta $A d e Q u a S$, que tem o objetivo de dar suporte às etapas do processo de avaliação de software. É baseado no Modelo Fuzzy de Avaliação de Qualidade de Software (MFAQS), para obter resultados mais reais sobre o grau de qualidade de atributos subjetivos através do julgamento de um grupo de especialistas. Além disso, é apresentado um exemplo de automatização do processo de avaliação de Especificações de Requisitos de Software (ERS).
\end{abstract}

Palavras-chave: Medições de Qualidade de Software, Modelos de Avaliação da Qualidade de Software, Ferramentas de Avaliação da Qualidade de Software.

\begin{abstract}
The software quality has had an increasing importance as a crucial factor to maintain the competitive level of the organizations. The software quality evaluation is indispensable in the quality obtainment and guarantee, both in a development process and in a final product.

This article presents the AdeQuaS tool, which has the objective of supporting the stages of software evaluation process. It is based on the Fuzzy Model to Software Quality Evaluation (FMSQE), in order to get effective results about the quality degree of subjective attributes through the judgment of a group of specialists. Besides, an example of the evaluation process automation to Software Requirement Specifications is presented.
\end{abstract}

Key-words: Software Quality Metrics, Software Quality Evaluation Models, Software Quality Evaluation Tools.

\section{Introdução}

A avaliação da qualidade de software tem sido um dos maiores alvos de pesquisa nos últimos anos, apesar de ainda possuir muitos aspectos a serem explorados. Tem como 
objetivo maior obter a garantia da qualidade, seja em um produto intermediário do processo de desenvolvimento ou produto final. Por este motivo, é fundamental adequá-la aos objetivos específicos da organização e do produto [4, 5, 6, 18, 21, 28, 29].

Muitas dificuldades surgem no processo de avaliação de um produto, que se iniciam no planejamento e na definição de como será realizada. Pode-se citar, como exemplo, a especificação de atributos de qualidade relevantes ao objeto de avaliação, definição de qual método de avaliação e medição é mais adequado, estabelecimento da combinação de métricas e medidas $[15,16,25,27,31,32,35]$, que quantifiquem adequadamente os atributos em resultados reais $[2,3,9,11,12,14,17,22,24,26]$.

Além disso, obter resultados quantitativos, que traduzam valores reais pela consolidação do julgamento de diversos avaliadores, com níveis de experiência e pontos de vista diferentes, para atributos subjetivos, é uma tarefa desafiadora [7, 8, 34].

Um método que tem minimizado estes problemas e obtido resultados satisfatórios em diversos trabalhos [1, 10,12] é o Modelo Fuzzy para Avaliação da Qualidade de Software (MFAQS) [8]. Esse modelo utiliza a robustez da teoria fuzzy para representação matemática sobre conceitos subjetivos através de operações de agregação.

Apesar do MFAQS ser flexível em várias situações e resolver de modo satisfatório o problema de agregação de medidas, sua utilização manual exige um enorme esforço. Neste trabalho, é proposta a ferramenta fuzzy AdeQuaS, baseada nesse modelo, que automatiza o processo de avaliação da qualidade, para se obter resultados mais eficientes e em um menor espaço de tempo.

Assim sendo, pretende-se contribuir para aumentar a viabilidade na execução de processos de avaliação de qualidade de software. Em face da crescente necessidade e busca pela qualidade e grande desejo de reduzir custos e prazos, a ferramenta fuzzy AdeQuaS propõe-se a suportar o processo de avaliação de software, promovendo mecanismos de investigação do nível de qualidade, através do julgamento de especialistas, como também a análise e a obtenção de resultados mais confiáveis. Este trabalho apresenta a aplicação da ferramenta fuzzy AdeQuaS na avaliação de qualidade de Especificação de Requisitos de Software (ERS) [13], já realizada e validada anteriormente.

Este artigo está organizado como se segue: a seção 2 fornece uma visão geral sobre a avaliação da qualidade de software, enfocando o modelo de qualidade e nas fases do processo. $\mathrm{Na}$ seção 3, são descritas as etapas do MFAQS e o funcionamento da ferramenta fuzzy AdeQuaS. A seção 4 refere-se ao estudo de caso, que apresenta os resultados obtidos nas avaliações realizadas. Na seção 5, as conclusões deste trabalho são apresentadas.

\section{Avaliação da Qualidade de Software}

O primeiro passo para a avaliação da qualidade de software é a determinação do modelo de qualidade $[19,20,23]$. Esta etapa corresponde à definição do conjunto de atributos relevantes a um determinado produto, que deverá ser adaptado adequadamente ao contexto da avaliação.

O modelo pode ser baseado na proposta fornecida pela ISO/IEC 9126 [19], que especifica as características e subcaracterísticas, que podem ser aplicadas a qualquer tipo de software. Estão apresentadas em duas partes: ( $i$ ) qualidade interna e externa; e (ii) qualidade em uso. A qualidade interna e externa referem-se ao funcionamento interno e ao 
comportamento dentro de um ambiente externo. Já a qualidade em uso corresponde ao seu contexto operacional, do ponto de vista do usuário.

A ISO/IEC 14598 [20,23] divide o processo de avaliação da qualidade de produto de software em quatro etapas principais: ( $i$ ) estabelecer os requisitos de avaliação; (ii) especificar a avaliação; (iii) projetar a avaliação; e (iv) executar a avaliação.

O estabelecimento dos requisitos de avaliação e especificação da avaliação englobam as atividades de definição de objetivos, objeto de avaliação, modelo de qualidade, métricas, medição e pontuação. O planejamento dos procedimentos e das responsabilidades no processo, dos métodos e dos cronogramas é a próxima etapa. Em seguida, a avaliação propriamente dita é executada.

O MFAQS [8] oferece suporte a todas as etapas do processo de avaliação, definido pela ISO/IEC 14598 [20]. Além disso, através da utilização da teoria fuzzy, possibilita tratar e analisar os dados obtidos de um grupo de especialistas, gerando o grau de consenso do julgamento dos avaliadores e o grau de qualidade de atributos utilizados na avaliação. A ferramenta fuzzy AdeQuaS, que será mostrada a seguir, automatiza esse modelo, enfocando sua utilização e suas funcionalidades peculiares mais importantes.

\section{AdeQuaS - Ferramenta Fuzzy para Avaliação da Qualidade de Software}

O MFAQS ou Modelo Rocha Estendido [8], automatizado pela ferramenta fuzzy AdeQuaS, engloba três situações distintas, descritas a seguir:

- Situação 1: determinação de um Padrão de Qualidade (PQ) de um produto ou domínio de aplicação;

- Situação 2: avaliação da qualidade de um produto de software, apoiando-se em um $P Q$ previamente definido;

- Situação 3: estimativa da qualidade de um produto de software, sem que haja um PQ já estabelecido.

No processo de construção da ferramenta fuzzy AdeQuaS, sentiu-se a necessidade de adaptar o MFAQS ao padrão ISO/IEC 9126 [19]. Esta adaptação foi realizada, segundo a estrutura apresentada na Figura 3.1.

- Características de qualidade: conjunto de atributos de um produto de software, através do qual sua qualidade é descrita e avaliada. Uma característica de qualidade de software pode ser detalhada em múltiplos níveis de subcaracterísticas. A subcaracterística de mais baixo nível (métrica) é chamada de subcaracterística primitiva e é passível de avaliação;

- Processos de avaliação: determinam o processo e os instrumentos a serem utilizados, de forma a se medir o grau de presença, no produto, de um determinado critério;

- Medidas: são os resultados da avaliação do produto, segundo os critérios, através de termos lingüísticos fuzzy, mapeados por números fuzzy;

- Medidas agregadas: são os resultados da agregação das medidas, obtidos ao se avaliar de acordo com os critérios. São, também, os resultados da agregação de critérios em subfatores, fatores, objetivos, e no valor final do produto de software;

- Funções Fuzzy: mapeiam os atributos de qualidade primitivos ou agregados, através do conjunto de termos lingüísticos estabelecidos, quantificando-os. 


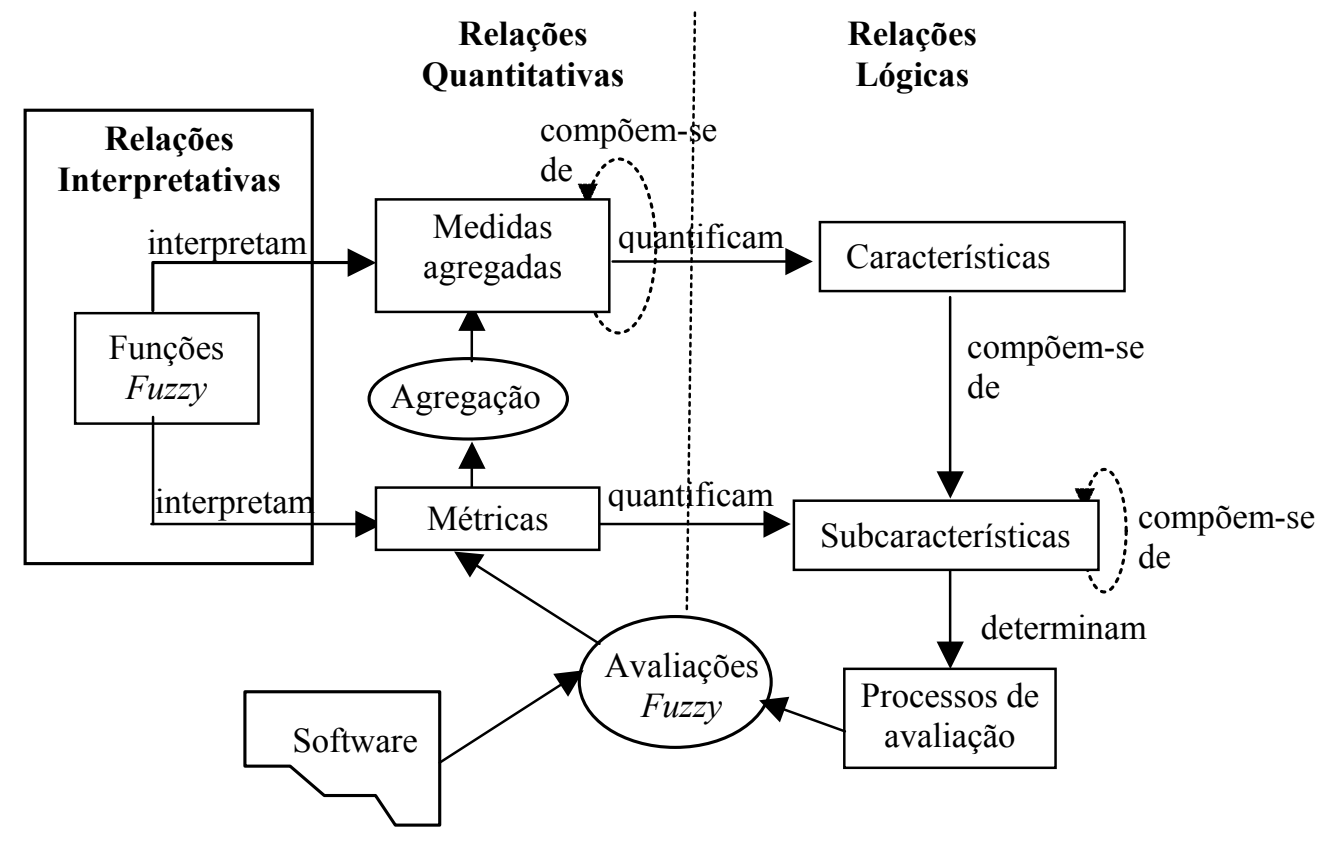

Figura 3.1. Modelo Fuzzy para Avaliação da Qualidade de Software

Portanto, ao se adaptar o MFAQS para a ISO/IEC 9126, este pode ser instanciado para o Modelo Rocha [33] original, pois ambos possuem, em sua essência, uma estrutura hierárquica de atributos de qualidade. Assim sendo, as características de qualidade do MFAQS podem ser instanciadas como sendo os objetivos de qualidade (nível hierárquico mais alto do Modelo Rocha). As subcaracterísticas de qualidade do MFAQS, por possuírem múltiplos níveis, podem ser instanciadas em fatores, subfatores e critérios, definidos no Modelo Rocha.

O MFAQS é constituído de cinco etapas, identificadas a seguir [8]:

3.1 Identificação do objeto a ser avaliado, do conjunto de atributos de qualidade de software a ser considerado na avaliação e das instituições pesquisadas;

3.2 Escolha dos especialistas: obtém o perfil do especialista (QIPE) e avalia a importância de seu julgamento dentro do processo.

3.3 Determinação do grau de importância de cada atributo de qualidade, identificado no item 3.1: os especialistas avaliam o grau em que o atributo está sendo satisfeito.

3.4 Tratamento dos dados coletados dos especialistas, na avaliação de cada atributo de qualidade considerado: obtém-se o grau de importância de cada atributo de qualidade estabelecido, através do peso dos especialistas e do coeficiente de consenso entre esses especialistas, pela aplicação de matrizes de similaridade fuzzy.

3.5 Agregação dos atributos de qualidade de software, em cada nível hierárquico do modelo de qualidade: os valores resultantes dos atributos obtidos na etapa anterior serão agregados, obtendo-se o resultado final das características e subcaracterísticas de qualidade requeridas.

Todas estas etapas foram implementadas na ferramenta fuzzy AdeQuaS [30], como mostrado na Figura 3.2. Para facilitar o processo de avaliação da qualidade, a ferramenta é composta por dois módulos: AdeQuas-Analisador e AdeQuaS-Avaliador.

O módulo AdeQuaS-Analisador é o módulo principal da ferramenta, no qual são realizadas as principais atividades do processo de avaliação da qualidade, tais como: (i) definição da avaliação e estabelecimento dos objetivos; (ii) identificação do objeto de 
avaliação; (iii) escolha e cadastro dos especialistas participantes do processo de avaliação; (iv) elaboração do Questionário de identificação do perfil do especialista (QIPE); (v) estabelecimento dos requisitos de qualidade, através da elaboração de um Questionário de avaliação (QA); (vi) acompanhamento da coleta de julgamentos dos especialistas; e (vii) geração de resultados dos dados coletados dos especialistas e da agregação de atributos.

O módulo AdeQuaS-Avaliador tem o objetivo de facilitar a pesquisa de campo, sendo utilizado pelo especialista participante da avaliação. É complementar e dependente do módulo principal, sendo um visualizador e um coletor de informações da avaliação. As tarefas realizadas pelo avaliador são: (i) visualizar as informações da avaliação e a definição do objeto a ser avaliado; (ii) conferir e corrigir seus próprios dados cadastrados no sistema; (iii) responder ao QIPE; e (iv) responder ao QA.

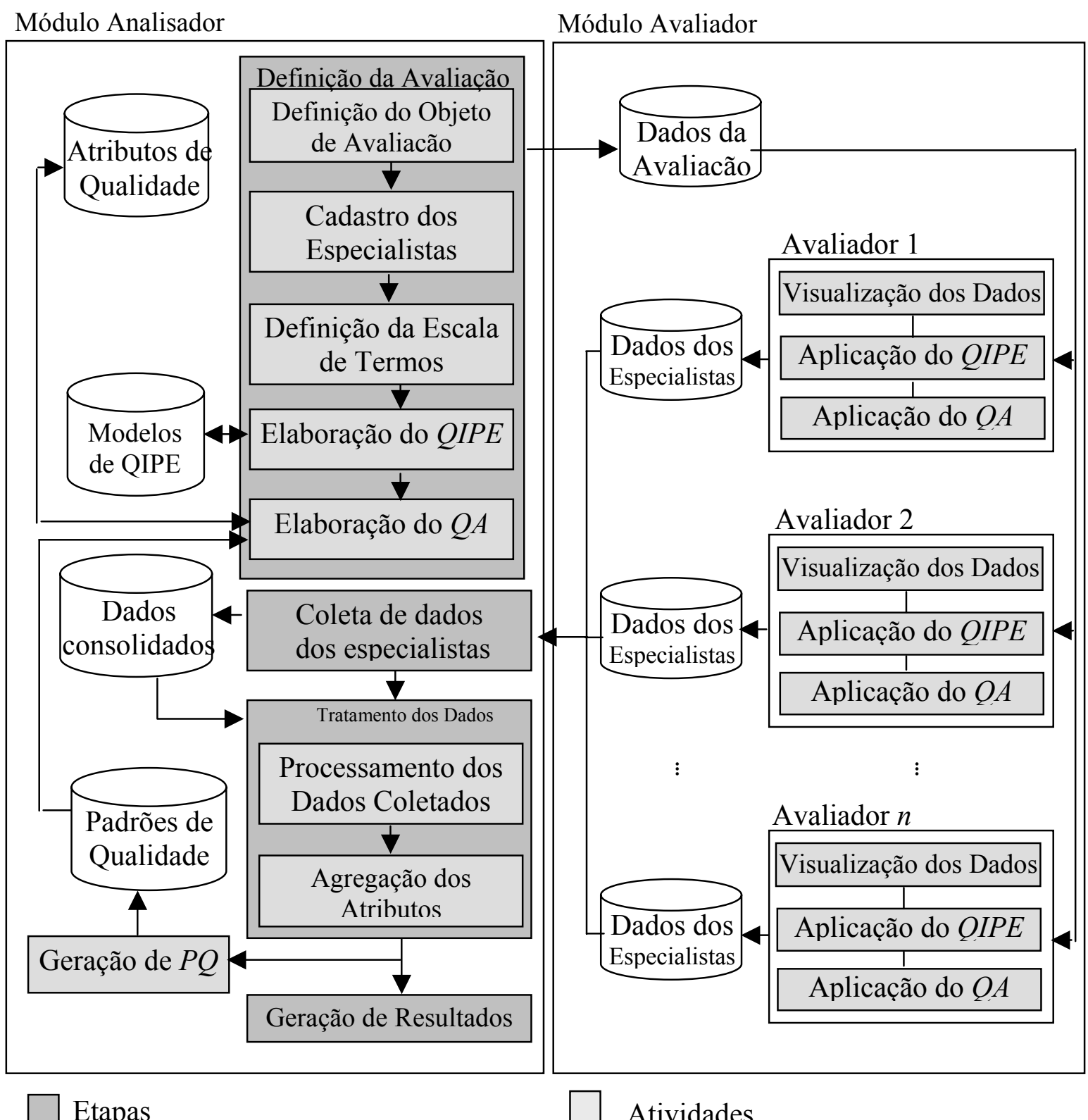

Figura 3.2 Arquitetura da ferramenta AdeQuaS 
O funcionamento da ferramenta fuzzy será mostrado com os dados referentes à avaliação da determinação do padrão de qualidade para Especificação de Requisitos de Software [13], que estão disponíveis em [8].

$\mathrm{Na}$ avaliação, os especialistas deram notas de 0 a 4 para cada atributos de qualidade, que passou pelo processo de "fuzificação", isto é, a transformação dessas notas em números fuzzy normais triangulares, conforme a Tabela 3.1.

Um número fuzzy normal triangular pode ser representado por $\tilde{N}(a, m, b)$, onde os valores $a$ e $b$ identificam, respectivamente, os limites inferior e superior da base do triângulo, onde o grau de pertinência $\mu_{\tilde{\mathrm{A}}}(x)=0$. O valor de $m$ corresponde à altura do triângulo, onde $\mu_{\tilde{\mathrm{A}}}(x)=1$.

Tabela 3.1 - Números fuzzy normais triangulares para a avaliação da qualidade [8]

\begin{tabular}{|c|l|l|l|}
\hline Nota & $\begin{array}{c}\text { Números Fuzzy } \\
\text { Triangulares }\end{array}$ & $\begin{array}{c}\text { Termo } \\
\text { Lingüístico }\end{array}$ & \multicolumn{1}{c|}{ Interpretação } \\
\hline $\mathbf{0}$ & $\tilde{N}_{1}=(0,0 ; 0,0 ; 1,0)$ & Sem relevância & O atributo de qualidade não tem importância. \\
\hline $\mathbf{1}$ & $\tilde{N}_{2}=(0,0 ; 1,0 ; 2,0)$ & Pouco relevante & O atributo de qualidade tem pouca importância. \\
\hline $\mathbf{2}$ & $\tilde{N}_{3}=(1,0 ; 2,0 ; 3,0)$ & Relevante & O atributo de qualidade é desejável. \\
\hline $\mathbf{3}$ & $\tilde{N}_{4}=(2,0 ; 3,0 ; 4,0)$ & Muito relevante & O atributo de qualidade é muito importante. \\
\hline $\mathbf{4}$ & $\tilde{N}_{5}=(3,0 ; 4,0 ; 4,0)$ & Imprescindível & O atributo de qualidade é imprescindível. \\
\hline
\end{tabular}

A definição da avaliação, realizada no módulo AdeQuaS-Analisador, deverá englobar todos os instrumentos necessários para a execução da avaliação, que são:

- informações sobre o objeto da avaliação (Figura 3.3);

- cadastro do grupo de especialistas, que irão participar do processo de avaliação (Figura 3.4);

- definição da escala de termos lingüísticos, que consiste na tabela de transformação do julgamento em números fuzzy triangulares, necessários nas etapas de agregação (Figura 3.5);

- elaboração do questionário de avaliação do perfil do avaliador (QIPE), que será utilizado para calcular o peso do especialista baseado em sua experiência profissional, como descrito na Etapa 3.2 do MFAQS (Figura 3.6); e

- elaboração do questionário de avaliação (QA), que consiste na árvore de atributos relevantes ao objeto de avaliação utilizada pelos avaliadores para realizar o julgamento do objeto em questão (Figura 3.7).

Após a avaliação estar completamente definida, os dados serão acoplados ao módulo AdeQuaS-Avaliador. Dependendo da disposição geográfica dos avaliadores, poderão existir vários módulos AdeQuaS-Avaliador, cada um disponível para um ou mais avaliadores.

Neste momento, a avaliação propriamente dita será executada. Os avaliadores poderão visualizar os dados referentes ao objeto da avaliação, semelhante à Figura 3.3. Os julgamentos poderão ser efetuados pela aplicação do QIPE (Figura 3.8) e aplicação do QA (Figura 3.9).

A aplicação do QA é composta pelas questões definidas nos processos de avaliação no momento da elaboração do QA (Figura 3.7) e os termos lingüísticos definidos (Figura 3.5). 


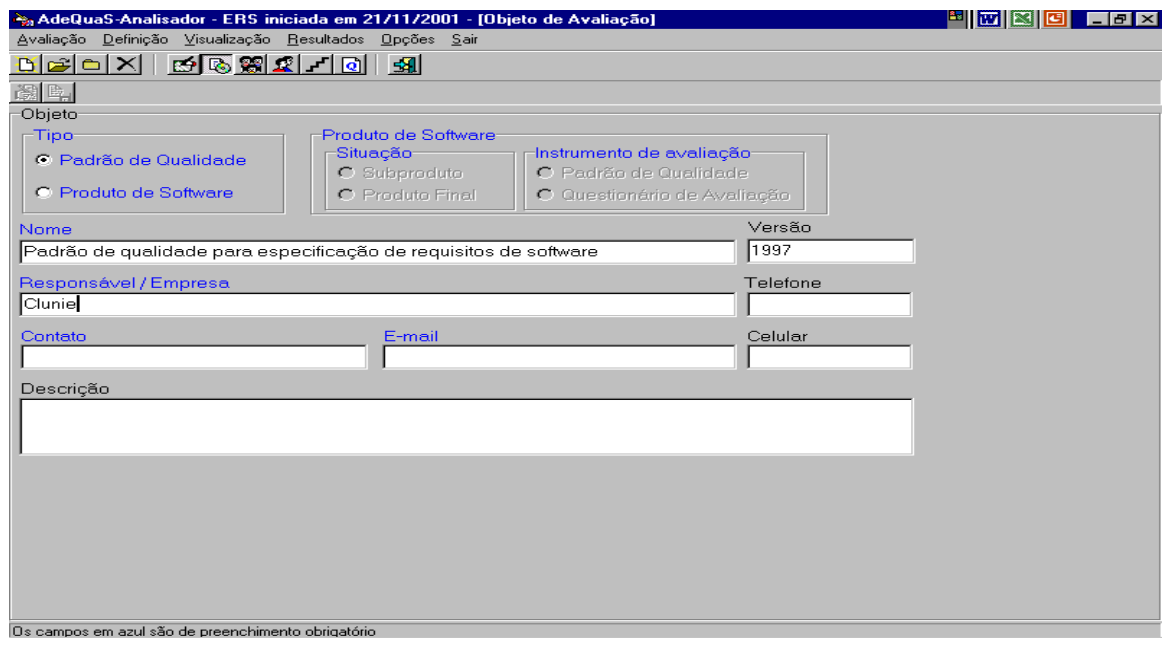

Figura 3.3 Definição do objeto a ser avaliado

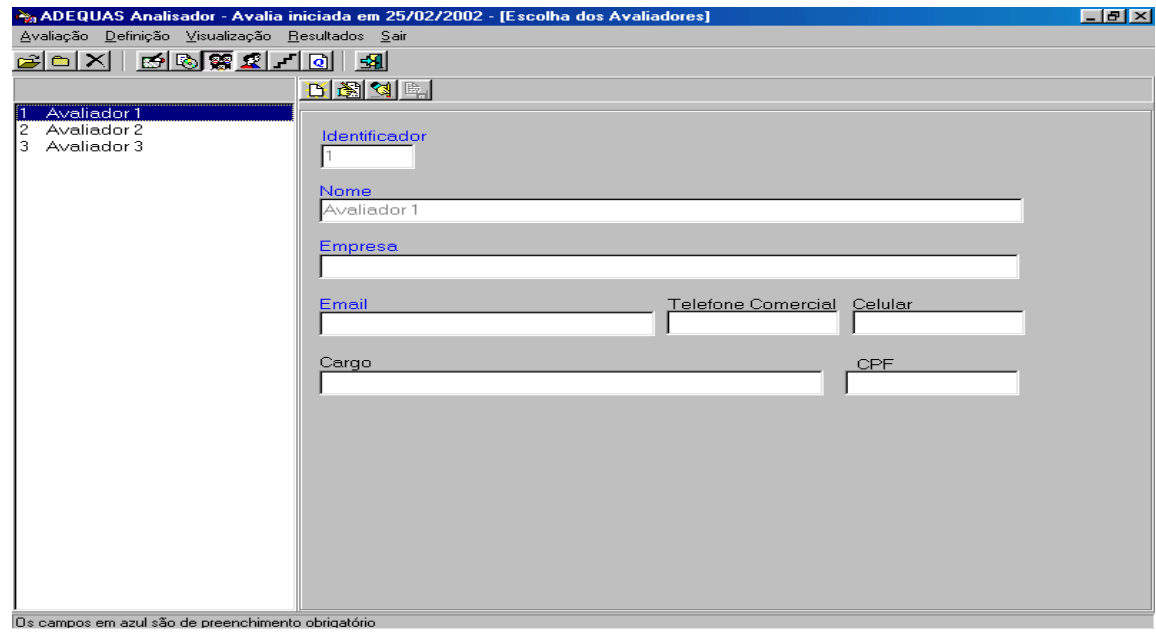

Figura 3.4 Cadastro do grupo de especialistas

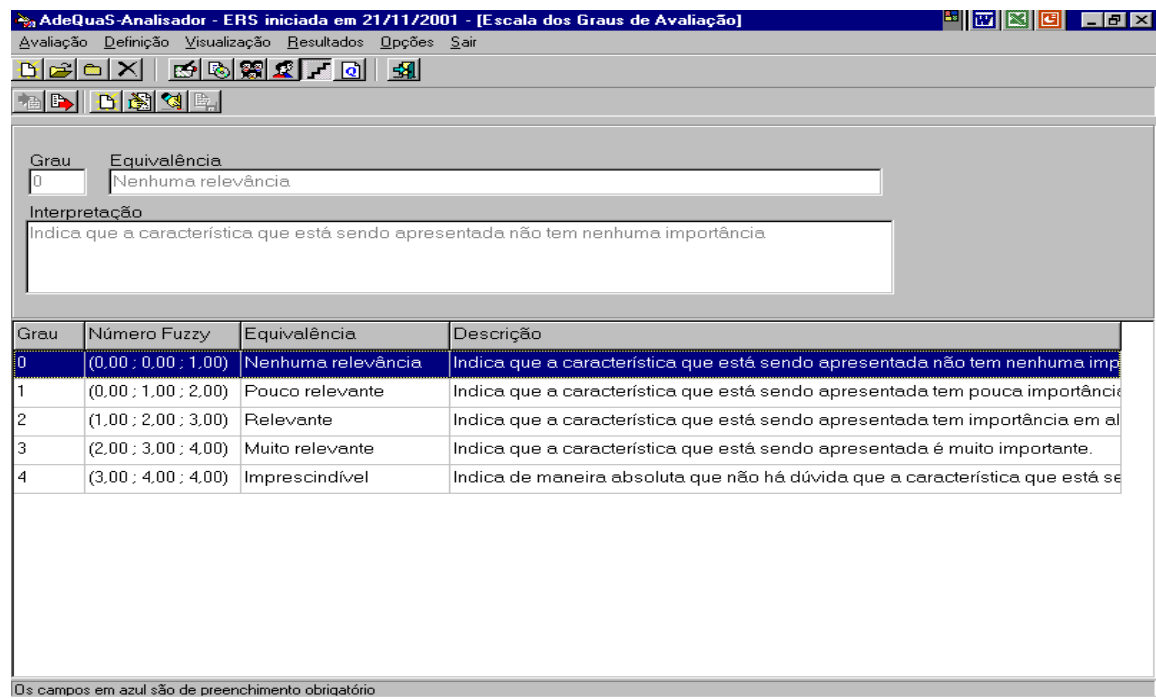

Figura 3.5 Definição da escala de termos lingüísticos (graus de avaliação) 


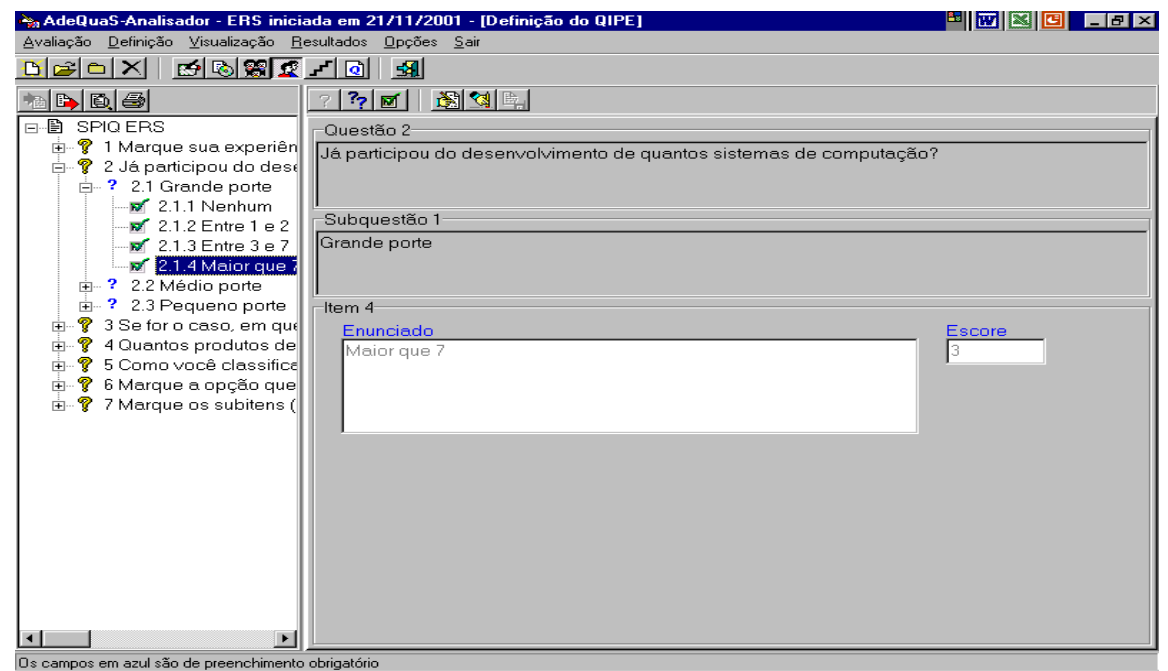

Figura 3.6 Elaboração do questionário de identificação do perfil do avaliador (QIPE)

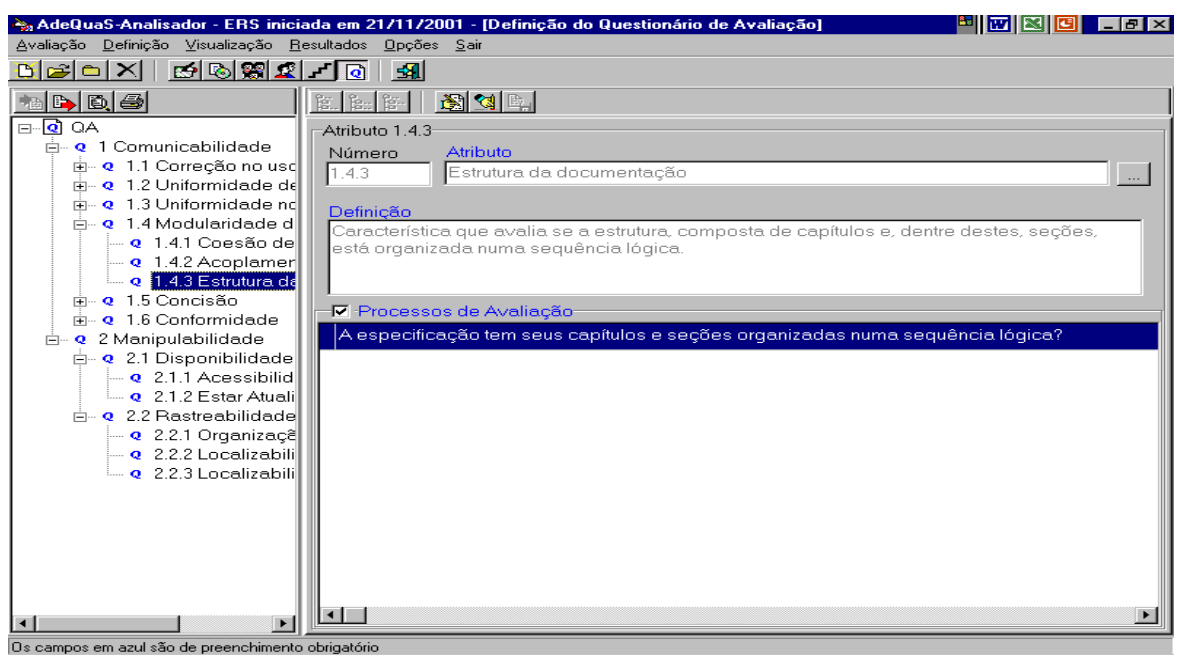

Figura 3.7 Elaboração do questionário de avaliação (QA)

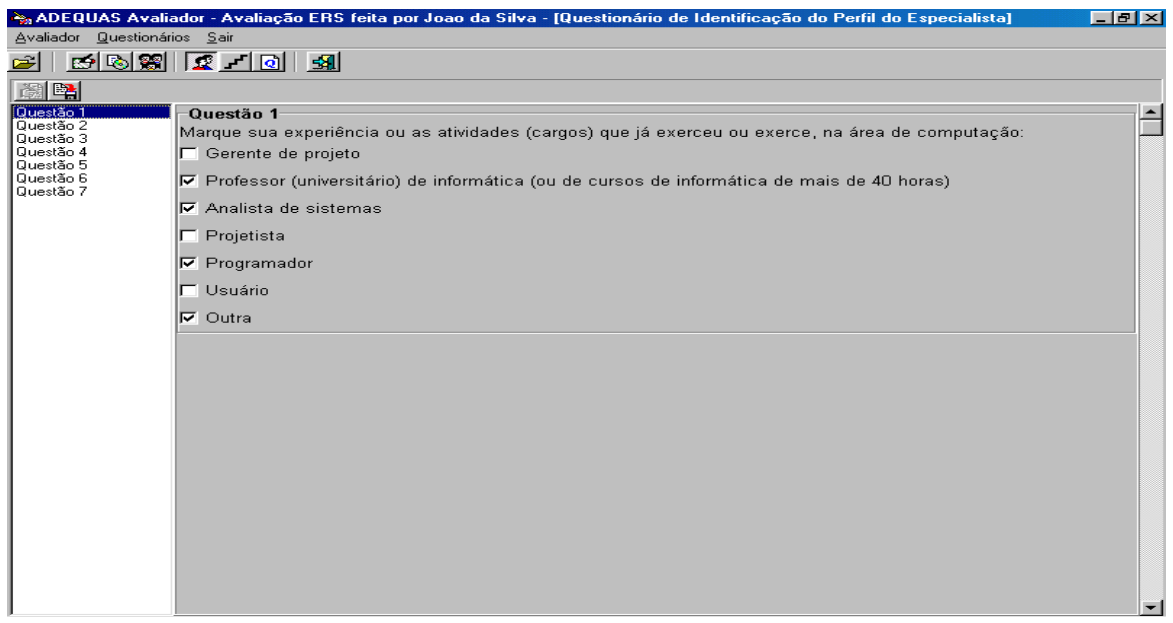

Figura 3.8 Aplicação do QIPE 


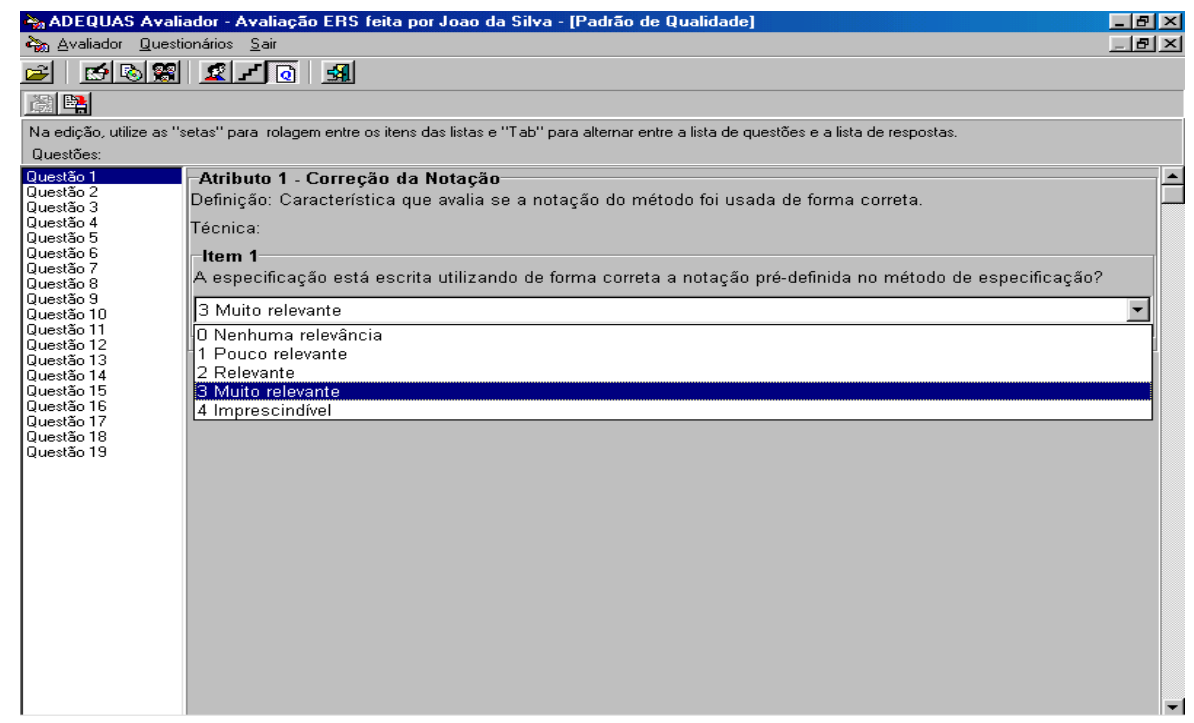

Figura 3.9 Aplicação do QA

\section{Estudo de Caso}

O estudo de caso realizado através da ferramenta fuzzy AdeQuaS [30] apresenta duas avaliações, que englobam duas situações previstas pelo MFAQS, descritas na Seção 3: (i) determinação do padrão de qualidade de um domínio de aplicação; e (ii) avaliação de um produto de software, apoiando-se em um PQ já previamente definido.

Ambas as avaliações foram resultados de pesquisas de campo já realizadas e validadas, apresentadas em [8], relacionadas à Especificação de Requisitos de Software (ERS) [13].

O questionário de identificação do perfil do especialista (QIPE), utilizado nas avaliações foi elaborado com sete questões, que englobam aspectos relativos à experiência profissional, grau de instrução e treinamento em computação.

\subsection{Determinação do padrão de qualidade para ERS}

Para realizar o levantamento do padrão de qualidade para ERS, a pesquisa foi realizada com 16 especialistas que estiveram diretamente envolvidos com elaboração de ERS. O perfil (valor individual apurado pelo QIPE) e o peso do especialista (valor relativo em relação ao grupo) são apresentados na Figura 4.1 em ordem decrescente de importância de julgamento de cada um.

Os atributos de qualidade para ERS definidos por Clunie [13] apresentam-se hierarquizados nos níveis de qualidade segundo o Modelo Rocha [33]: objetivos, fatores, subfatores e critérios. A avaliação apresentada neste artigo abrange um subconjunto de fatores e subfatores, que corresponde ao objetivo Confiabilidade da Representação, e está apresentada na Tabela 4.1.

O resultado apresentado pela ferramenta aparece na Figura 4.2, incluindo a interpretação dos fatores e subfatores definidos, obtidos na etapa de agregação do MFAQS. 
Esses resultados apresentam-se bastantes semelhantes aos da avaliação da qualidade de ERS, realizada sem a utilização da ferramenta.

Analisando-se o resultado apresentado, é possível observar que o subfator Disponibilidade aparece com o maior valor do termo lingüístico imprescindivel da escala definida na Figura 3.5, sendo 4,5\% muito relevante e 95,5\% imprescindivel. Portanto, em ERS, este atributo é o mais importante.

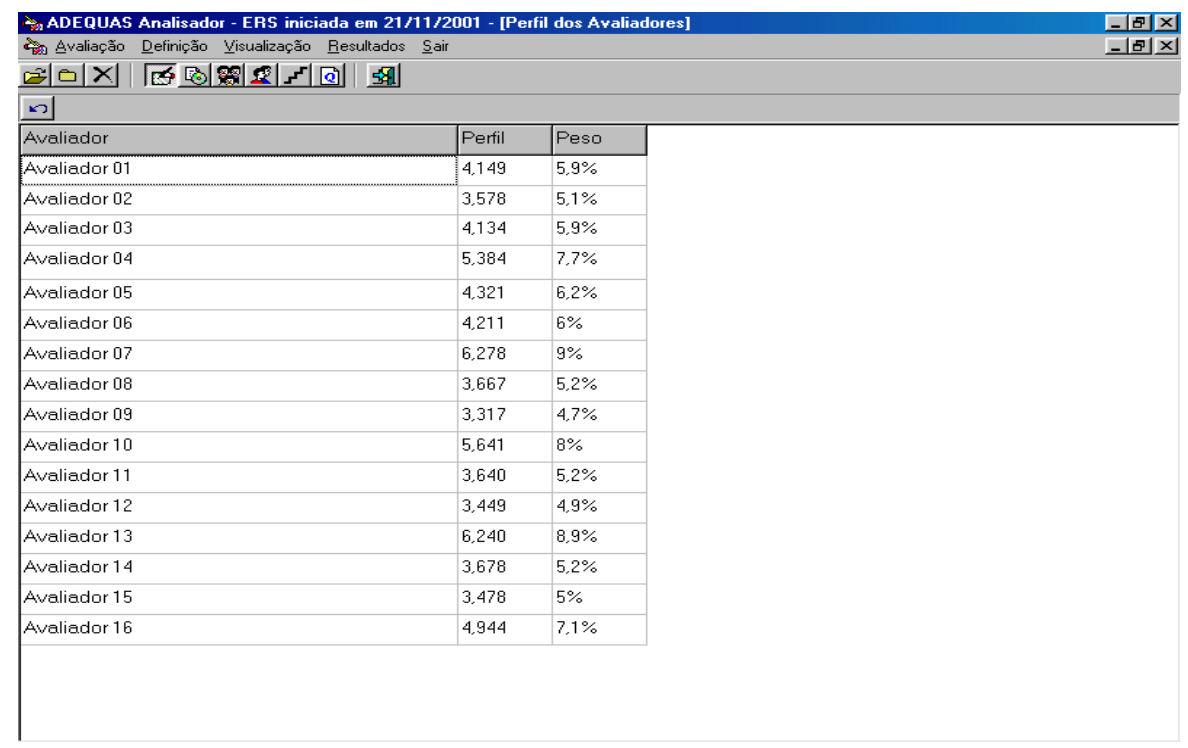

Figura 4.1 Resultado o QIPE na avaliação de padrão de qualidade ERS.

Tabela 4.1 Avaliação de atributos de qualidade para ERS [8]

\begin{tabular}{|l|c|}
\hline Atributos de Qualidade de Software para ERS & PQ para ERS \\
\hline Fator: Comunicabilidade & $\tilde{N}=(2,39 ; 3,39 ; 3,91)$ \\
\hline Subfator: Correção no uso do método & $\tilde{N}=(2,47 ; 3,47 ; 3,94)$ \\
\hline Subfator: Uniformidade de Terminologia & $\tilde{N}=(2,66 ; 3,66 ; 4,00)$ \\
\hline Subfator: Uniformidade no nível de abstração & $\tilde{N}=(1,55 ; 2,55 ; 3,48)$ \\
\hline Subfator: Modularidade da documentação & $\tilde{N}=(2,36 ; 3,36 ; 3,97)$ \\
\hline Subfator: Concisão & $\tilde{N}=(2,55 ; 3,55 ; 3,86)$ \\
\hline Subfator: Conformidade & $\tilde{N}=(2,41 ; 3,41 ; 3,97)$ \\
\hline Fator: Manipulabilidade & $\tilde{N}=(2,75 ; 3,75 ; 3,98)$ \\
\hline Subfator: Disponibilidade & $\tilde{N}=(2,95 ; 3,95 ; 4,00)$ \\
\hline Subfator: Rastreabilidade & $\tilde{N}=(2,56 ; 3,56 ; 3,96)$ \\
\hline
\end{tabular}




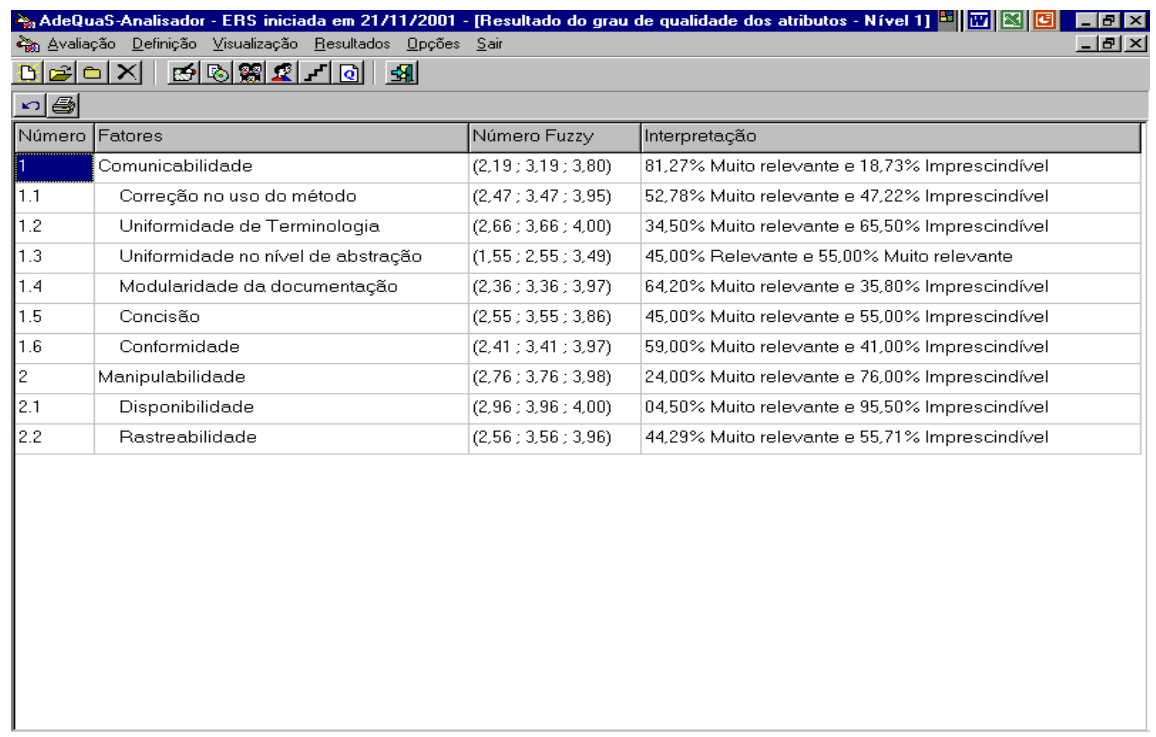

Figura 4.2 Visualização do resultado dos atributos para ERS

\subsection{Avaliação de uma ERS real}

Os dados referentes à esta etapa do estudo de caso foram obtidos em [8] através da avaliação da modelagem de dados do módulo financeiro do sistema SIGAH-multimídia. Um grupo de 3 especialistas foram envolvidos, respondendo um questionário de avaliação composta por um subconjunto dos atributos de qualidade de ERS. O resultado dos pesos dos especialistas está mostrado na Figura 4.3.

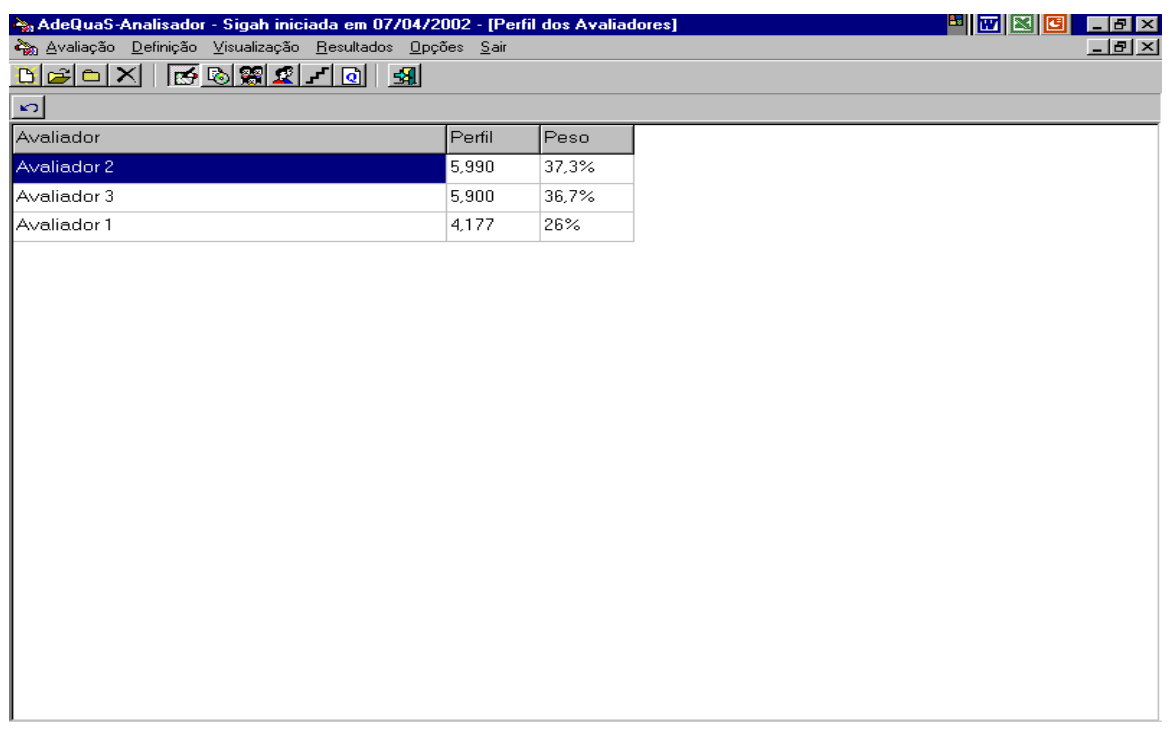

Figura 4.3 Resultado do QIPE na avaliação de uma ERS real.

Diferentemente da avaliação anterior, por ser uma avaliação de produto de software, os resultados obtidos foram confrontados com os valores do padrão de qualidade gerado na 
Subseção 4.1 e apresentados os índices de qualidade, que indicam o quanto o produto de software atingiu a qualidade necessária para cada atributo considerado (Figura 4.4).

$\mathrm{Na}$ Tabela 4.2, são apresentados os valores dos atributos que foram avaliados, relevantes ao contexto desta avaliação.

Tabela 4.2 Avaliação de atributos de qualidade para ERS [8]

\begin{tabular}{|l|c|}
\hline Atributos de Qualidade de Software para ERS & Números Fuzzy \\
\hline Fator: Comunicabilidade &.-- - \\
\hline Subfator: Correção do Uso do Método & $\tilde{N}=(3,00 ; 4,00 ; 4,00)$ \\
\hline Subfator: Uniformidade de Terminologia & $\tilde{N}=(2,17 ; 3,17 ; 3,75)$ \\
\hline Subfator: Uniformidade no Nível de Abstração & $\tilde{N}=(2,35 ; 3,35 ; 4,00)$ \\
\hline Subfator: Concisão & $\tilde{N}=(2,00 ; 3,00 ; 4,00)$ \\
\hline Fator: Manipulabilidade & $-\ldots-$ - \\
\hline Subfator: Disponibilidade & $\tilde{N}=(1,46 ; 2,46 ; 3,46)$ \\
\hline Subfator: Rastreabilidade & $-\ldots$ \\
\hline
\end{tabular}

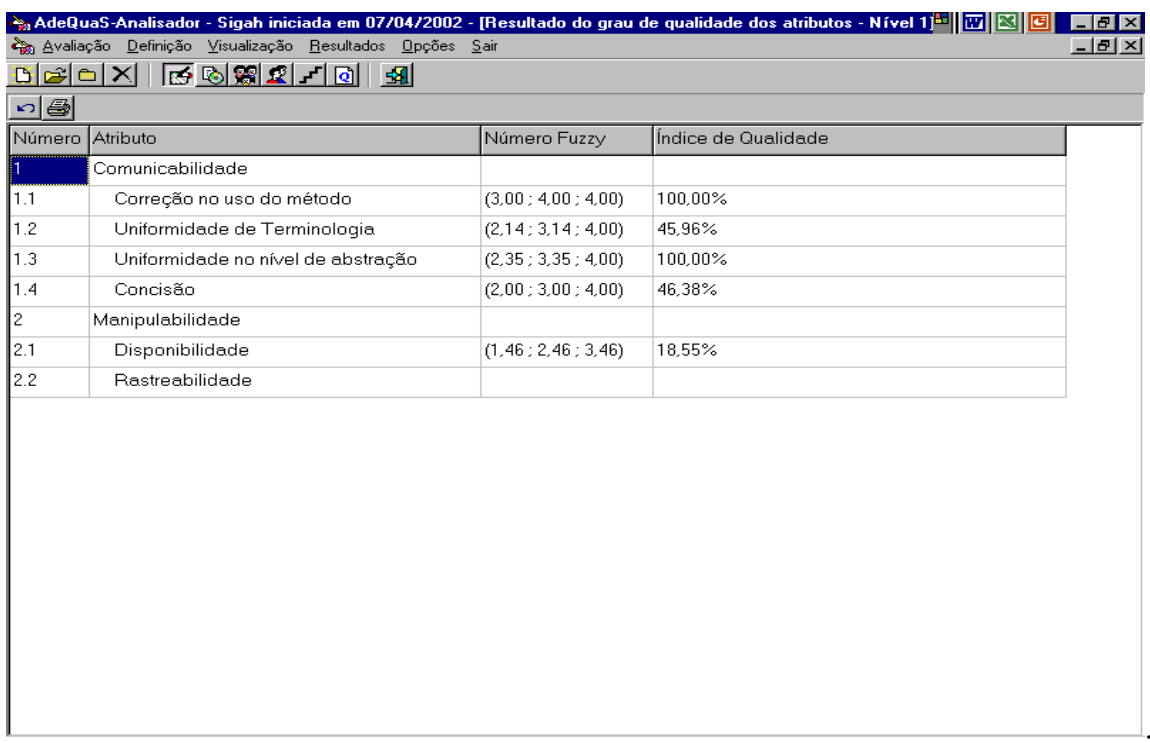

Figura 4.4 Visualização do resultado de uma avaliação de ERS

Segundo os índices de qualidade apresentados para a especificação do produto SIGAHMultimídia, pode-se inferir que os subfatores Correção no uso do método e Uniformidade no nível de abstração atingiram $100 \%$ da qualidade necessária para o produto, porém, o subfator mais importante Disponibilidade atingiu apenas $18,55 \%$, que é bem abaixo do que realmente é esperado para uma ERS. 


\section{Conclusão}

Neste trabalho, desenvolveu-se a ferramenta fuzzy AdeQuaS com a finalidade de viabilizar a utilização do MFAQS, através de um processo automatizado, fornecendo suporte ao processo de avaliação da qualidade de software.

Pode-se elencar como principais contribuições deste trabalho, os seguintes itens:

- Adaptação do MFAQS (ou Modelo Rocha Estendido) ao padrão ISO/IEC 9126;

- Desenvolvimento da ferramenta fuzzy AdeQuaS, que implementa o MFAQS já no padrão ISO, minimizando de sobremaneira o esforço na realização de um processo de avaliação da qualidade, gerando os resultados da avaliação de forma imediata e automática;

- Utilização de múltiplos níveis hierárquicos de atributos de qualidade (quantos forem necessários), que podem ser nomeados e identificados, segundo a nomenclatura desejada ou utilizada em trabalhos de qualidade anteriores;

Além dos itens acima, a ferramenta fuzzy AdeQuaS apresenta as seguintes funcionalidade adicionais:

- Geração de padrões de qualidade (PQ) associados a um domínio de aplicação, segundo uma taxonomia de domínios de aplicação, que podem ser armazenados no banco de dados da ferramenta, e podem ser reutilizados em avaliações de qualidade posteriores;

- Reutilização dos Questionários de identificação do perfil do especialista (QIPE) e do Questionário de Avaliação (QA), armazenados no banco de dados da ferramenta, adequando-os às peculiaridades do processo de avaliação em curso;

\section{Referências Bibliográficas}

[1] Albuquerque, A.B., 2001, Qualidade de websites de comércio eletrônico, Dissertação de Mestrado, Centro de Ciências Tecnológicas, Universidade de Fortaleza, Fortaleza, CE.

[2] Antonioni, J. A. R., Braga, N., 1995, Qualidade em software: manual de aplicação da ISO-9000, São Paulo, Makron Books.

[3] Arthur, L. J., 1994, Melhorando a qualidade de software: um guia para o TQM, Rio de Janeiro, Infobook.

[4] Basili, V. R., 1992, Software Modeling and Measurement: the goal/question/metric paradigm, Technical Report CS-TR-2956, University of Maryland

[5] Basili, V. R., 1994, The goal question metric approach, Encyclopedia of Software Engineering, University of Maryland, site acessado em maio/2002: http://www.cs.toronto.edu/ sme/CSC444F/handouts/GQM-paper.pdf.

[6] Bassman, M. J. et al., 1995, Software Measurement Guidebook - Revision 1, Software Engineering Laboratory Series, National Aeronautics and Space Administration (NASA), Maryland.

[7] Belchior, A. D. et al., 1996, Agregação de atributos de qualidade de software usando-se a teoria fuzzy, X Simpósio Brasileiro de Engenharia de Software - Workshop de Quaildade, São Paulo.

[8] Belchior, A. D., 1997, Um modelo fuzzy para Avaliação da Qualidade de Software, Tese de Doutorado, Departamento de Engenharia de Sistemas e Computação - COPPE, Universidade Federal do Rio de Janeiro, RJ. 
[9] Blaschek, J. R. S., 1996, Metodologia para avaliação de sistemas de informação, Seleção de textos, Centro Internacional de Tecnologia de Software, p. 109-124.

[10] Branco Jr., E. C., Belchior, A. C. 2001, Processos gerenciais de projetos de software: uma abordagem qualitativa, VIII Workshop de Qualidade de Software, Rio de Janeiro.

[11] Camargo Jr., J. B. et al., 1997, O uso de fatores de qualidade na avaliação da segurança de software em sistemas críticos, VIII Conferência Internacional de Tecnologia de Software, Curitiba.

[12] Campos, F. et al, 1998, Qualidade de software agropecuário: uma visão do usuário, IX Conferência Internacional de Tecnologia de Software, Curitiba.

[13] Clunie, C. E., 1997, Avaliação da Qualidade de Especificações Orientadas a Objeto, Tese de Doutorado, Departamento de Engenharia de Sistemas e Computação - COPPE, Universidade Federal do Rio de Janeiro.

[14] Costa, M. C. C. et al, 1997, Elaboração de um diagnóstico para um projeto de avaliação e melhoria da qualidade de software, VIII CITS, Curitiba.

[15] Duarte, K. C. Falbo, R. A., 2000, Uma ontologia de qualidade de software. Workshop de Qualidade de Software, João Pessoa.

[16] Fenton, N. E., Pfleeger, S. L., 1997, Software metrics: a rigorous and practical approach, Second edition, Thompson Computers.

[17] Gladcheff, A. P., Sanches, R., Silva, D. M., 2001, Um instrumento de avaliação de qualidade de software educacional: como elaborá-lo, Workshop de Qualidade de Software, Rio de Janeiro.

[18] Gomes, A. et al., 2000, Medição e melhoria de processos de software, Workshop de Qualidade de Software, João Pessoa.

[19] ISO/IEC 9126-1, 2001, Software engineering - Product quality - Part 1: quality model.

[20] ISO/IEC 14598-1, 1998, Information technology - software product evaluation - Part 1: general overview.

[21] Kirner, T. G., Abib, J. C., 1997, Uso da abordagem goal-question-metric na avaliação de qualidade de requisitos de software, Workshop de Qualidade de Software, Fortaleza.

[22] Kitchenham, B. et. al., 1996, Software Quality: the elusive target, IEEE Software, p. 1221.

[23] Koscianski, A. et. al., 1999, Guia para utilização das normas sobre avaliação de qualidade de produto de software - ISO/IEC 9126 E ISO/IEC 14598, ABNT, Curitiba.

[24] Lemes, M. J. R., Fernandes, C. T., 1997a, Concepção de plano de garantia da qualidade de software. VIII Conferência Internacional de Tecnologia de Software, Curitiba.

[25] Lemes, M. J. R., Fernandes, C. T., 1997b, Uma taxonomia para métricas de software. XI Simpósio Brasileiro de Engenharia de Software - Workshop de Qualidade de Software, Fortaleza.

[26] Losavio, F., Chirinos, L., 1999, Evaluación de la calidad en el desarrollo de sistemas interactivos. X CITS: Curitiba.

[27] Martins, A. V., 1996, Métricas de software em modelos de dados, Seleção de textos, Centro Internacional de Tecnologia de Software, p. 89-107.

[28] McFeeley, R., 1996, IDEAL: a user's guide for software process improvement, Handbook CMU/SEI-96-HB-001, Software Engineering Institute, Pittsburgh.

[29] Möller, K. H., 1993, Software metrics: a practitioner's guide to improved product development, Chapman \& Hall Computing, London. 
[30] Oliveira, K. R., 2001, Software quality evaluation: a practice approach using AdeQuaS Fuzzy tool, 24 ${ }^{\text {th }}$ International Conference on Software Engineering - Workshop on Software Quality, Orlando.

[31] Pfleeger, S. L., 1998 Software Engineering: theory and practice, Prentice Hall, Nova Jersey.

[32] Pressman, R. S., 2000, Software engineering: a practitioner's approach, Fifth edition, McGraw Hill, Nova York.

[33] Rocha, A. R. C., 1983, Um modelo para avaliação da qualidade de especificações, Tese de Doutorado, Departamento de Engenharia de Software e Ciências da Computação, Pontifícia Universidade Católica (PUC), Rio de Janeiro, RJ.

[34] Rocha, A. R. C., et.al., 2001, Qualidade de Software: Teoria e Prática, São Paulo, Prentice Hall.

[35] Valle, A. et al., 2001, Mensuração e Análise: os programas existentes satisfazem nossas necessidades?, XII Conferência Internacional de Tecnologia de Software, Curitiba. 